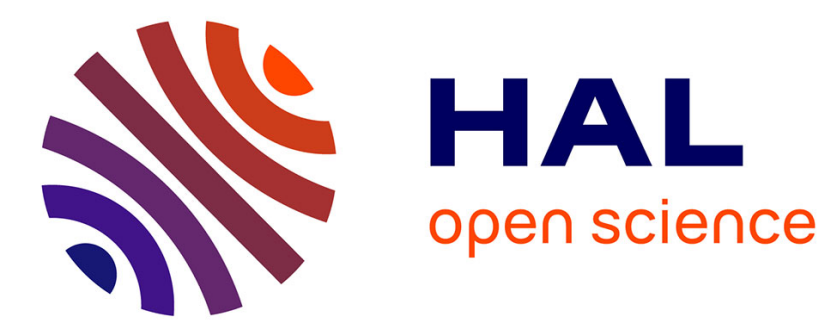

\title{
Physiological models comparison for the analysis of ASL FMRI data
}

\author{
Aina Frau-Pascual, Florence Forbes, Philippe Ciuciu
}

\section{To cite this version:}

Aina Frau-Pascual, Florence Forbes, Philippe Ciuciu. Physiological models comparison for the analysis of ASL FMRI data. 12th IEEE International Symposium on Biomedical Imaging, ISBI 2015, Apr 2015, New York, United States. pp.1348-1351, 10.1109/ISBI.2015.7164125 . hal-01249014

\section{HAL Id: hal-01249014 https://hal.science/hal-01249014}

Submitted on 7 Jan 2016

HAL is a multi-disciplinary open access archive for the deposit and dissemination of scientific research documents, whether they are published or not. The documents may come from teaching and research institutions in France or abroad, or from public or private research centers.
L'archive ouverte pluridisciplinaire HAL, est destinée au dépôt et à la diffusion de documents scientifiques de niveau recherche, publiés ou non, émanant des établissements d'enseignement et de recherche français ou étrangers, des laboratoires publics ou privés. 


\title{
PHYSIOLOGICAL MODELS COMPARISON FOR THE ANALYSIS OF ASL FMRI DATA
}

\author{
Aina Frau-Pascual ${ }^{(1,2)} \quad$ Florence Forbes $^{(1)} \quad$ Philippe Ciuciu $^{(2,3)}$ \\ (1) INRIA, MISTIS, Grenoble University, LJK, Grenoble, France \\ ${ }^{(2)}$ INRIA, Parietal, F-91893 Orsay, France \\ ${ }^{(3)} \mathrm{CEA} / \mathrm{DSV} / \mathrm{I}^{2} \mathrm{BM}$ NeuroSpin center, Bât. 145, F-91191 Gif-sur-Yvette, France
}

\begin{abstract}
Physiological models have been proposed to describe the processes that underlie the link between neural and hemodynamic activity in the brain. Among these, the Balloon model describes the changes in blood flow, blood volume and oxygen concentration when an hemodynamic response is ensuing neural activation. Next, an hemodynamic model links these variables to the blood-oxygen-leveldependent (BOLD) effect. Taken together, these equations allow the precise modeling of the coupling between the cerebral blood flow and hemodynamic response. However, several competing versions of the hemodynamic model and different physiological parameters values have been described in the literature. In this work, we analyse Arterial Spin Labelling (ASL) functional Magnetic Resonance Imaging (fMRI) data, which contains both perfusion and hemodynamic effects, to compare the impact of different settings in the coupling between blood flow and hemodynamic response.
\end{abstract}

Index Terms - fMRI, ASL, Balloon model, CBF, JDE

\section{INTRODUCTION}

In the past decade, physiological models have been described to explain the physiological changes caused by neural activity. In [1-3] different models have been introduced: neural coupling, which maps neural activity to ensuing cerebral blood flow (CBF); the Balloon model, which relates $\mathrm{CBF}$ to volume and deoxyhemoglobin changes, and hemodynamic model, also referred to as BM in [4], that relates these parameters to the blood-oxygen-level-dependent (BOLD) effect. These models thus provide a complete description of the physiological process underlying hemodynamic activity, from neural activation to the hemodynamics or BOLD effect measurement. However, different parameter settings have been proposed in the Balloon model formulation and several BM have been presented in [4]. Such variability provides flexibility to model physiological responses but also introduces more complexity.

The Arterial Spin Labelling (ASL) signal embodies two components: an hemodynamic or BOLD component and a perfusion one. The ASL signal comes from a T2* image acquisition with successive alternate control/tag settings, with inversed magnetization in the tag image. An hemodynamic or BOLD effect can be found in both control/tag images, while a perfusion effect can be captured from the control-tag difference. Their typical shapes are respectively described by the hemodynamic response function, here referred as BRF for BOLD response function, and the perfusion response function (PRF). These two response functions can be estimated using the probabilistic joint detection-estimation (JDE) formalism [5, 6], although the PRF estimation remains difficult because of the noisier nature of the perfusion component within the ASL signal. For this reason, in [7] we used a physiological link between PRF and BRF shapes as a prior knowledge in the JDE framework $[5,6]$. However, the physiological parameters we chose and the BM model we used in [7] were not completely in accordance with the analysis performed in [4], where the performance of the different models was compared. Hence, in this paper we want to replicate the analysis of [4] but for ASL data and to identify which model outperforms the other for informing the link between perfusion and hemodynamics (PRF/BRF link) in the JDE analysis of ASL data. Results on real data could give us a hint on the best set of parameters to use in the Balloon and hemodynamic (BM) models.

The rest of the paper is organized as follows: the models considered in the computation of the perfusion/hemodynamics link are presented and analysed in Section 2; the physiologically informed ASL JDE model is introduced in Section 3; and some results are presented and discussed in Sections 4-5.

\section{A PHYSIOLOGICAL LINK BETWEEN PERFUSION AND HEMODYNAMICS}

An approximate relationship between the perfusion and hemodynamic response functions can be derived from physiological models. In this section, we recall the work presented in [7], in which the extended Balloon model and the BM model were used to describe a link between perfusion and hemodynamic response functions. Following the same reasoning as in [4], we further analyse the different models to recover a more accurate perfusion/hemodynamics link, with the correct set of parameters.

\subsection{The extended Balloon model}

The Balloon model was first proposed in [1] to link neuronal and vascular processes by considering the capillary as a balloon that inflates under the effect of blood flow variations. More specifically, the model describes how, after some stimulation, the local blood flow $\boldsymbol{f}_{\text {in }}(t)$ increases and leads to the subsequent augmentation of the local capillary volume $\boldsymbol{\nu}(t)$. This incoming blood is strongly oxygenated but only part of the oxygen is consumed. It follows a local decrease of the deoxyhemoglobin concentration $\boldsymbol{\xi}(t)$ and therefore a BOLD effect variation. The Balloon model was then extended in [2] to include the effect of the neuronal activity $\boldsymbol{u}(t)$ on the variation of some auto-regulated flow inducing signal $\boldsymbol{\psi}(t)$ so as to eventually link neuronal to hemodynamic activity. The global physiological model corresponds then to a nonlinear system with four state variables $\left\{\boldsymbol{\psi}, \boldsymbol{f}_{i n}, \boldsymbol{\nu}, \boldsymbol{\xi}\right\}$ corresponding to normalized flow inducing signal, local blood flow, local capillary volume, and deoxyhemoglobin concentration. Their interactions over time are described by the following system of nonlinear differential equations: 


$$
\left\{\begin{aligned}
\frac{d \boldsymbol{f}_{i n}(t)}{d t} & =\boldsymbol{\psi}(t) \\
\frac{d \boldsymbol{\psi}(t)}{d t} & =\eta \boldsymbol{u}(t)-\frac{\boldsymbol{\psi}(t)}{\tau_{\psi}}-\frac{\boldsymbol{f}_{i n}(t)-1}{\tau_{f}} \\
\frac{d \boldsymbol{\xi}(t)}{d t} & =\frac{1}{\tau_{m}}\left(\boldsymbol{f}_{i n}(t) \frac{1-\left(1-E_{0}\right)^{1 / \boldsymbol{f}_{i n}}(t)}{E_{0}}-\boldsymbol{\xi}(t) \boldsymbol{\nu}(t)^{\frac{1}{\tilde{\tilde{w}}}-1}\right) \\
\frac{d \boldsymbol{\nu}(t)}{d t} & =\frac{1}{\tau_{m}}\left(\boldsymbol{f}_{i n}(t)-\boldsymbol{\nu}(t)^{\frac{1}{\tilde{w}}}\right)
\end{aligned}\right.
$$

with initial conditions $\boldsymbol{\psi}(0)=0, \boldsymbol{f}_{\text {in }}(0)=\boldsymbol{\nu}(0)=\boldsymbol{\xi}(0)=1$. Lower case notation is used for normalized functions by convention. The system depends on 5 hemodynamic parameters: $\tau_{\psi}, \tau_{f}$ and $\tau_{m}$ are time constants respectively for signal decay/elimination, auto-regulatory feedback from blood flow and mean transit time, $\tilde{w}$ reflects the ability of the vein to eject blood, and $E_{0}$ is the oxygen extraction fraction. Another parameter $\eta$ is the neuronal efficacy weighting term that models neuronal efficacy variability.

\subsection{The hemodynamic model}

Buxton et al [3] proposed the following expression to link the hemodynamic response (BRF) ${ }^{1} \boldsymbol{h}(t)$ to physiological quantities considering the intra-vascular and extra-vascular components:

$$
\boldsymbol{h}(t)=V_{0}\left[k_{1}(1-\boldsymbol{\xi}(t))+k_{2}\left(1-\frac{\boldsymbol{\xi}(t)}{\boldsymbol{\nu}(t)}\right)+k_{3}(1-\boldsymbol{\nu}(t))\right]
$$

where $k_{1}, k_{2}$ and $k_{3}$ are scanner-dependent constants and $V_{0}$ is the resting blood volume fraction. This equation can be linearized into:

$$
\boldsymbol{h}(t)=V_{0}\left[\left(k_{1}+k_{2}\right)(1-\boldsymbol{\xi}(t))+\left(k_{3}-k_{2}\right)(1-\boldsymbol{\nu}(t))\right] .
$$

As synthesized in [4], where the hemodynamic model is referred to as $\mathrm{BM}$, different expressions were proposed for $k_{1}, k_{2}$ and $k_{3}$ : the classical ones (classical BM) in [3], and their revised (revised BM) version in [8]. Hereafter, we will use the same notation as Stephan et al [4]: CBM and RBM stand for models using the classical and revised expressions, respectively, and subscripts " $\mathrm{L}$ " and " $\mathrm{N}$ " for the nonlinear (Eq. (1)) and linear (Eq. (2)) expressions:

\section{CBM}

\section{RBM}

$$
\begin{array}{ll}
k_{1}=\left(1-V_{0}\right) 4.3 \vartheta_{0} E_{0} T E & k_{1}=4.3 \vartheta_{0} E_{0} T E \\
k_{2}=2 E_{0} & k_{2}=\epsilon r_{0} E_{0} T E \\
k_{3}=1-\epsilon & k_{3}=1-\epsilon
\end{array}
$$

where $\vartheta_{0}$ is the frequency offset at the outer surface of the magnetized vessel for fully deoxygenated blood, $r_{0}$ the slope of the relation between intra-vascular relaxation rate and oxygen saturation, and $\epsilon$ the ratio of intra- and extravascular signal.

In the end, we have different combinations: classical linear $\mathrm{BM}\left(C B M_{L}\right)$, revised linear $\mathrm{BM}\left(R B M_{L}\right)$, classical nonlinear $\mathrm{BM}\left(C B M_{N}\right)$ and revised nonlinear $\mathrm{BM}\left(R B M_{N}\right)$. Different values have been proposed in [2,9] (see Tab. 1) for the physiological and $\mathrm{BM}$ parameters, and we consider some of them in this paper. According to [10], we also considered at 3T: $r_{0}=100 \mathrm{~s}^{-1}$ and $\vartheta_{0}=80.6 s^{-1}$. For $\epsilon$ parameter, the values given by [4] have been used: $0.4,1$ and 1.43 .

\subsection{Physiological linear relationship between response func- tions}

Akin to [7], starting from the system of differential equations, we derive an approximate relationship between the PRF, namely $\boldsymbol{g}(t)$ and the BRF, namely $\boldsymbol{h}(t)$. Both PRF and BRF are percent signal

\footnotetext{
${ }^{1}$ To clarify, the hemodynamic response corresponds to the impulse response, namely the BRF, only when a single stimulus or neural event is considered as input
}

Table 1. Physiological and BM parameters used in [2,9].

\begin{tabular}{|c|c|c|c|c|c|c|c|}
\hline & $\eta$ & $\tau_{\psi}$ & $\tau_{f}$ & $\tau_{m}$ & $\tilde{w}$ & $E_{0}$ & $V_{0}$ \\
\hline$[2]$ & 0.5 & 1.25 & 2.5 & 1 & 0.2 & 0.8 & 0.02 \\
\hline$[9]$ & 0.54 & 1.54 & 2.46 & 0.98 & 0.33 & 0.34 & 1 \\
\hline
\end{tabular}

changes. We consider $\boldsymbol{g}(t)=\boldsymbol{f}_{\text {in }}(t)-1$, as $\boldsymbol{f}_{\text {in }}(t)$ is the normalized perfusion, with initial value 1 . Therefore the state variables become $\{\boldsymbol{\psi}, \boldsymbol{g}, 1-\boldsymbol{\nu}, 1-\boldsymbol{\xi}\}$. In what follows, we will drop the time index $t$ and consider functions $\boldsymbol{h}, \boldsymbol{\psi}$, etc. in their discretized vector form.

By linearizing the system of differential equations around the resting point $\{\boldsymbol{\psi}, \boldsymbol{g}, 1-\boldsymbol{\nu}, 1-\boldsymbol{\xi}\}=\{\mathbf{0}, \mathbf{0}, \mathbf{0}, \mathbf{0}\}$ as in [9], we get:

$$
\left\{\begin{array}{l}
\boldsymbol{D} \boldsymbol{g}=-\boldsymbol{\psi} \\
\left(\boldsymbol{D}+\frac{\boldsymbol{I}}{\tilde{w} \tau_{m}}\right)(1-\boldsymbol{\nu})=-\frac{1}{\tau_{m}} \boldsymbol{g} \\
\left(\boldsymbol{D}+\frac{\boldsymbol{I}}{\tau_{m}}\right)(1-\boldsymbol{\xi})=-\left(\gamma \boldsymbol{I}-\frac{1-\tilde{w}}{\tilde{w} \tau_{m}^{2}}\left(\boldsymbol{D}+\frac{\boldsymbol{I}}{\tilde{w} \tau_{m}}\right)^{-1}\right) \boldsymbol{g}
\end{array},\right.
$$

where $\boldsymbol{D}$ and $\boldsymbol{I}$ are the first order differential operator and the identity matrix respectively, and $\gamma=\frac{1}{\tau_{m}}\left(1+\frac{\left(1-E_{0}\right) \ln \left(1-E_{0}\right)}{E_{0}}\right)$.

By considering this system of equations and the BM equations (linear (2)/nonlinear (1) forms), we can derive a linear relationship between $\boldsymbol{h}$ and $\boldsymbol{g}$ that reads $\boldsymbol{g}=\boldsymbol{\Omega} \boldsymbol{h}$ where:

$$
\boldsymbol{\Omega}=V_{0}^{-1}\left(\left(k_{1}+k_{2}\right) \boldsymbol{B}+\left(k_{3}-k_{2}\right) \boldsymbol{A}\right)^{-1}
$$

when Eq. (2) holds and

$$
\boldsymbol{\Omega}=V_{0}^{-1}\left(k_{1} \boldsymbol{B}+k_{2}(\boldsymbol{B}-\boldsymbol{A})(\boldsymbol{I}-\boldsymbol{A})^{-1}+k_{3} \boldsymbol{A}\right)^{-1}
$$

with

$$
\left\{\begin{array}{l}
\boldsymbol{A}=-\frac{1}{\tau_{m}}\left(\boldsymbol{D}+\frac{\boldsymbol{I}}{\tilde{w} \tau_{m}}\right)^{-1} \\
\boldsymbol{B}=-\left(\boldsymbol{D}+\frac{\boldsymbol{I}}{\tau_{m}}\right)^{-1}\left(\gamma \boldsymbol{I}-\frac{1-\tilde{w}}{\tilde{w} \tau_{m}^{2}}\left(\boldsymbol{D}+\frac{\boldsymbol{I}}{\tilde{w} \tau_{m}}\right)^{-1}\right) .
\end{array}\right.
$$

when Eq. (1) holds instead. Hence, we have different $\Omega$ matrices depending on the Balloon model parameters (see Tab. 1), the classical or revised expression for $k_{1}, k_{2}$ and $k_{3}$ (see Eqs. (3)-(5)), directly impacted by $\epsilon$ parameter, and the model (Eqs. (6)-(7)). Changing $\boldsymbol{\Omega}$ might therefore affect the PRF and BRF estimation results from ASL data and identifying the best combination of these ingredients is the issue we want to address in the present paper.

\subsection{Perfusion/hemodynamics link analysis on simulated data}

As we have seen, matrix $\Omega$ will vary depending on which model we consider: $\mathrm{CBM}_{L}, \mathrm{RBM}_{L}, \mathrm{CBM}_{N}$ and $\mathrm{RBM}_{N}$ with different possible values for $\epsilon$, and on which set of parameters we use: [2] or [9]. Here, we simulate a PRF by applying $\boldsymbol{g}_{m}=\boldsymbol{\Omega}_{m} \boldsymbol{h}_{\text {can }}$ to the canonical BRF shape ( $\boldsymbol{h}_{\text {can }}$ ) using different $\boldsymbol{\Omega}_{m}$ ( $m$ coding the model that we use) to find out which factors have a stronger impact on $\Omega$. As there is no canonical PRF, we use the canonical BRF as a reference.

Fig. 1(a) illustrates the cases for which we observed the strongest variability in the simulated PRF shape when applying $\boldsymbol{g}_{m}=\boldsymbol{\Omega}_{m} \boldsymbol{h}$ when $\boldsymbol{\Omega}_{m}$ is defined either by (6) or (7) using the parameters proposed in [9]. Besides, we also found variability between the generated PRFs associated with different $\epsilon$ values. Fig. 1(b) shows this fact for the $C B M_{L}$ model.

To draw significant conclusions about the statistical significance of the measured differences between PRF and canonical BRF, for each $\epsilon$ value we performed a 2 way-ANOVA including the model type $\left(C B M_{L}, R B M_{L}, C B M_{N}\right.$ and $\left.R B M_{N}\right)$ and the setting of 
(a)
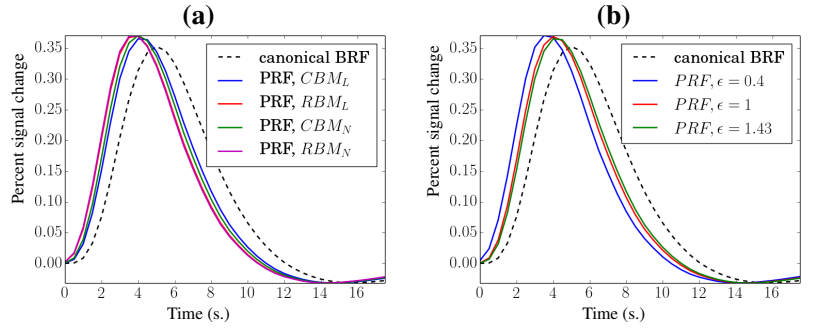

Fig. 1. PRFs resulting from $\boldsymbol{\Omega}_{m} \boldsymbol{h}_{\text {can }}$ when using model $m$, for parameters in [9] (a), and for the $C B M_{L}$ model (b).

parameters (see Tab. 1) as the two factors of interest. We entered the squared differences between the canonical BRF and the different PRFs as observations in our analysis. For $\epsilon=0.4$ only, we identified a very significant difference between the sets of parameters, but none between classical and revised or linear and nonlinear BM models at a 0.01 level of significance (F-test: $F=38.98$, p-val $<10^{-4}$ ). This result is also confirmed by the discrepancy depicted in Fig. 1(b) between the blue curve and the other traces. To sum up, the setting of physiological parameters and $\epsilon$ can impact the quality of the link between perfusion and hemodynamic response functions, whereas choosing a particular BM model has a limited influence. In what follows, we address the same concern on real ASL data acquired along an fMRI experiment.

\section{BAYESIAN HIERARCHICAL MODEL FOR ASL DATA ANALYSIS}

\subsection{ASL signal modeling}

The ASL JDE model described in [5, 6] considers functional homogeneous parcels with constant response shapes. In a given parcel $\mathcal{P}$, the generative model for ASL time series, with $M$ experimental conditions, reads $\forall j \in \mathcal{P},|\mathcal{P}|=J$ :

$$
\boldsymbol{y}_{j}=\sum_{m=1}^{M} \underbrace{a_{j}^{m} \boldsymbol{X}^{m} \boldsymbol{h}}_{(a)}+\underbrace{c_{j}^{m} \boldsymbol{W} \boldsymbol{X}^{m} \boldsymbol{g}}_{(b)}+\underbrace{\alpha_{j} \boldsymbol{w}}_{(c)}+\underbrace{\boldsymbol{P} \boldsymbol{\ell}_{j}}_{(d)}+\underbrace{\boldsymbol{b}_{j}}_{(e)}
$$

The measured signal $\boldsymbol{y}_{j}$ is decomposed into the components:

(a) Task-related hemodynamic component, where $\boldsymbol{h}$ is the unknown BRF shape and $\left\{a_{j}^{m}, j \in \mathcal{P}, m=1: M\right\}$ are the magnitude of activation or hemodynamic response levels (BRLs).

(b) Task-related perfusion component, that represents the variation of the perfusion around baseline when there is task-related activity. $\boldsymbol{g}$ is the PRF shape and $\left\{c_{j}^{m}, j \in \mathcal{P}, m=1: M\right\}$ are the magnitude of activation or perfusion response levels (PRLs).

(a-b) Task-related components. $\boldsymbol{X}$ encodes the lagged onset stimuli. The BRLs and PRLs (resp., $a_{j}^{m}$ and $c_{j}^{m}$ ) are assumed to follow $2 M$ different spatial Gaussian mixture models but these Gaussian variables are governed by $M$ common binary hidden Markov random fields $\left\{q_{j}^{m}, j \in \mathcal{P}, m=1: M\right\}$ encoding voxels' activation states for each experimental condition $m$ and promoting spatial correlation as in $[5,6]$.

(c) Perfusion baseline completes the modelling of the perfusion component and it is encoded by the scalar $\alpha_{j}$.

(b-c) The control/tag vector $\boldsymbol{w}=[1 / 2,-1 / 2,1 / 2, \ldots]$ and matrix $\boldsymbol{W}=\operatorname{diag}(\boldsymbol{w})$ encode the difference in magnetization signs between the consecutive and alternated control (positive magnetization) and tagged (negative magnetization) ASL volumes. (a) Auditory cortex results
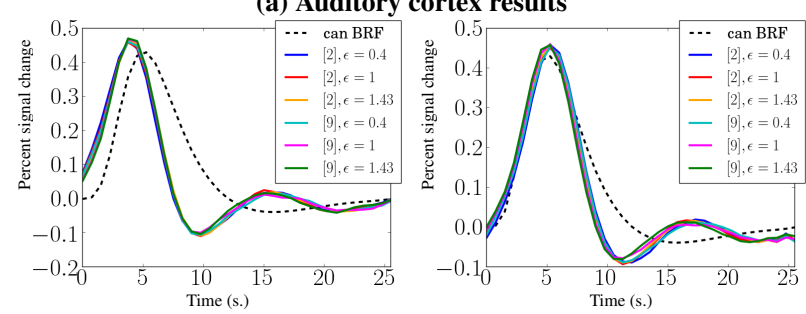

(b) Visual cortex results

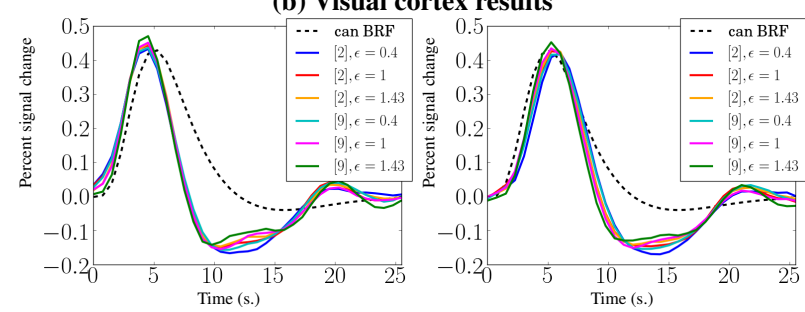

Fig. 2. PRF (left) and BRF (right) estimates for model $R B M_{N}$ with parameters in [2] and [9], considering different $\epsilon$ values, estimated in auditory (a) and visual (b) cortices.

(d-e) Drift and noise terms allow to account for a potential drift and any other nuisance effect. Noise is assumed to be white Gaussian, which is a tenable assumption in ASL compared to BOLD fMRI.

\subsection{A physiologically informed inference procedure}

In Eq. (8), the hemodynamic component can be estimated from the ASL data with an increased confidence compared to the perfusion component, as it is known to have a higher contrast-to-noise ratio (CNR). The relationship $\boldsymbol{g}=\boldsymbol{\Omega} \boldsymbol{h}$, which was already derived between both components in [7], was then used to a priori constrain the PRF from the BRF in a stochastic manner. Since the hemodynamic component is associated with a better CNR, it makes more sense to recover first the BRF $\boldsymbol{h}$ and to improve estimation of the PRF $\boldsymbol{g}$ through the following conditional distribution in the JDE formulation: $p(\boldsymbol{g} \mid \boldsymbol{h})=\mathcal{N}\left(\Omega \boldsymbol{h}, v_{\boldsymbol{g}} \boldsymbol{\Sigma}_{g}\right)$, where $\boldsymbol{\Sigma}_{g}=(\Delta t)^{4}\left(\boldsymbol{D}_{2}^{t} \boldsymbol{D}_{2}\right)^{-1}$ and $\boldsymbol{D}_{2}$ is the truncated second-order finite difference matrix that enforces temporal smoothness as a function of the prior variance $v_{\boldsymbol{g}}$ : see $[5,6]$ for details. As regards the BRF $\boldsymbol{h}$, as in previous works $[11,12]$, its prior distribution follows a centered multivariate Gaussian distribution: $\mathcal{N}\left(\mathbf{0}, v_{\boldsymbol{h}} \boldsymbol{\Sigma}_{h}\right)$ where $\boldsymbol{\Sigma}_{h}=\boldsymbol{\Sigma}_{g}$ and $v_{\boldsymbol{h}}$ is a free variance parameter.

By using this physiological prior, we are enforcing the relationship between the two response functions, and this allows a better perfusion component estimation. Next, as in [7], this relationship is used in a fully Bayesian JDE approach for ASL data where each fully conditional posterior distribution is sampled at a time in order to compute minimum mean square error (or posterior mean) estimators as regards the PRF and BRF shapes as well as the PRLs and BRLs and maximum a posteriori (MAP) estimators for detecting which voxels elicit evoked activity. The interested reader is invited to refer to [7] for details on the hybrid Gibbs-Metropolis algorithm.

\section{REAL DATA RESULTS}

Here, we performed ASL JDE analysis on ASL fMRI data, considering the different models and parameter settings for $\boldsymbol{\Omega}$ explored in Section 2. The analysis was performed on 8 individuals, although the results are shown for 1 subject only. The same conclusions hold for the other subjects. 


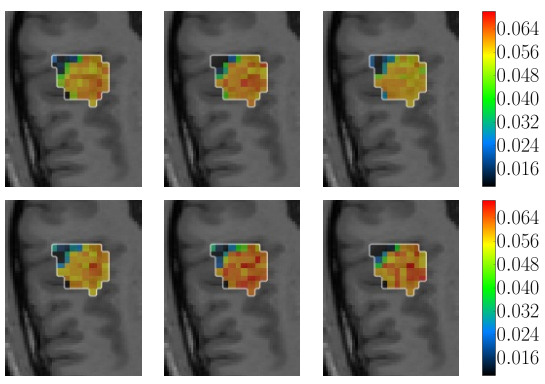

Fig. 3. Auditory cortex PRLs for model $R B M_{N}$ with parameters: [2] (top), [9] (bottom), considering $\epsilon$ (left to right) $0.4,1$ and 1.43.

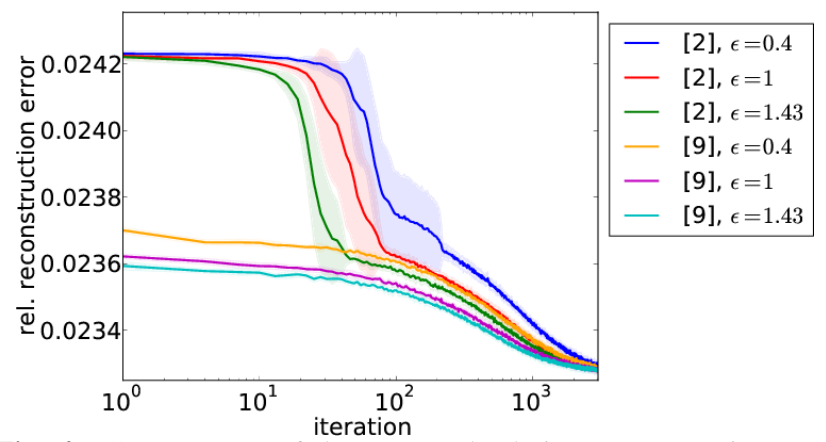

Fig. 4. Convergence of the averaged relative reconstruction error over 10 runs for the auditory cortex and model $R B M_{N}$. Standard deviations are shown with shaded colors.

The ASL data were recorded during an experiment specifically designed to map auditory and visual primary cortices, with 291 scans, repetition time $T R=3 \mathrm{~s}$, echo time $T E=18 \mathrm{~ms}$, FoV $192 \mathrm{~mm}$, dimensions $64 \times 64 \times 22$ voxels (resolution of $3 \times 3 \times 3.5$ $\mathrm{mm}^{3}$ ). The tagging scheme used was PICORE Q2T, with $T I_{1}=$ $700 \mathrm{~ms}, T I_{2}=1700 \mathrm{~ms}$. A fast event-related paradigm (mean $I S I=5.1 \mathrm{~s}$ ) was used, comprising sixty auditory and visual stimuli. Two regions of interest in the right temporal and left occipital lobes were defined manually as parcels of interest for looking at the evoked response in the the auditory and visual cortices, respectively.

Fig. 2 shows the perfusion and hemodynamic response functions estimated using different $\boldsymbol{\Omega}$ matrices for $p(\boldsymbol{g} \mid \boldsymbol{h})$ in the ASL JDE inference, considering $3000 \mathrm{MCMC}$ iterations. Here, the two parameter sets [2,9] were tested and $\boldsymbol{\Omega}$ was computed using the above mentioned models and $\epsilon$ values. Fig. 2 also depicts the canonical BRF (dashed line), which is in accordance with the BRF estimates for both methods. We observed very similar shapes, as well as similar perfusion response levels in Fig. 3 for the auditory cortex using $R B M_{N}$. A variability in PRF could impact the PRLs retrieved.

Fig. 4 shows the convergence of the relative reconstruction error (the lower the better) over MCMC iterations for the different parameter settings. BM models are not all shown as they have similar convergence speed. Interestingly, we observed a stronger variability between the two parameter sets as compared to changing the $\epsilon$ value. Important results are the lower relative reconstruction error of the parameters proposed in [9] from the first iteration, and the better performance in both sets of parameters ( [9] and [2]) for $\epsilon=1.43$. For this reason, we can consider the combination $[\epsilon=1.43$ and parameters as in [9]] as the one performing the best and offering promising perspectives. However, after 3000 iterations, the algorithm converges to good parameter estimates in all cases.

\section{DISCUSSION AND CONCLUSION}

A physiological link has been described, combining the Balloon and BM models, to achieve a better estimation of parameters in an ASL JDE framework. Different versions of the BM model have been described in the literature, and different parameter settings for the Balloon model have also been proposed. In this paper, we considered them altogether to assess their impact in the context of ASL data analysis. On simulated data, the selection of physiological parameters used in the Balloon model as well as the setting of $\epsilon$, were more critical than that of the BM model itself. On real ASL data, we confirmed this finding with a faster convergence in the joint estimation of perfusion and hemodynamic components of the signal, at least in the auditory and visual regions. Future work will be dedicated to confirm or infirm these results on other brain regions (eg, motor) and other data sets.

\section{Acknowledgements}

We would like to thank Salma Bougacha for insightful comments and interesting discussions.

\section{REFERENCES}

[1] R. B. Buxton, E. C. Wong, and L. R. Frank, "Dynamics of blood flow and oxygenation changes during brain activation: the balloon model," Magnetic Resonance in Medicine, vol. 39, pp. 855-864, 1998.

[2] K. J. Friston, A. Mechelli, R. Turner, and C. J. Price, "Nonlinear responses in fMRI: the balloon model, Volterra kernels, and other hemodynamics," Neuroimage, vol. 12, pp. 466-477, 2000.

[3] R. B. Buxton, K. Uludağ, D. J. Dubowitz, and T. T. Liu, "Modeling the hemodynamic response to brain activation," Neuroimage, vol. 23, pp. S220-S233, 2004.

[4] K. E. Stephan, N. Weiskopf, P. M. Drysdale, P. A. Robinson, and K. J. Friston, "Comparing hemodynamic models with DCM," Neuroimage, vol. 38, no. 3, pp. 387-401, 2007.

[5] T. Vincent, F. Forbes, and P. Ciuciu, "Bayesian BOLD and perfusion source separation and deconvolution from functional ASL imaging," in 38th Proc. IEEE ICASSP, Vancouver, Canada, 2013, pp. 1003-1007.

[6] T. Vincent, J. Warnking, M. Villien, A. Krainik, P. Ciuciu, and F. Forbes, "Bayesian joint detection-estimation of cerebral vasoreactivity from ASL fMRI data," in 16th Proc. MICCAI, LNCS Springer Verlag, Nagoya, Japan, 2013, vol. 2, pp. 616-623.

[7] A. Frau-Pascual, T. Vincent, J. Sloboda, P. Ciuciu, and F. Forbes, "Physiologically informed Bayesian analysis of ASL fMRI data," in Bayesian and grAphical Models for Biomedical Imaging, M.J. Cardoso, I. Simpson, T. Arbel, D. Precup, and A. Ribbens, Eds., vol. 8677 of LNCS, pp. 37-48. Springer, 2014.

[8] T. Obata, T. T. Liu, K. L. Miller, W.-M. Luh, E. C. Wong, L. R. Frank, and R. B. Buxton, "Discrepancies between BOLD and flow dynamics in primary and supplementary motor areas: application of the balloon model to the interpretation of BOLD transients," Neuroimage, vol. 21, no. 1, pp. 144-153, 2004.

[9] I. Khalidov, J. Fadili, F. Lazeyras, D. Van De Ville, and M. Unser, "Activelets: Wavelets for sparse representation of hemodynamic responses," Signal Processing, vol. 91, no. 12, pp. 2810-2821, 2011.

[10] Y. Behzadi and T.T. Liu, "An arteriolar compliance model of the cerebral blood flow response to neural stimulus," Neuroimage, vol. 25, no. 4, pp. 1100-1111, 2005.

[11] P. Ciuciu, J.-B. Poline, G. Marrelec, J. Idier, Ch. Pallier, and H. Benali, "Unsupervised robust non-parametric estimation of the hemodynamic response function for any fMRI experiment," IEEE Trans. on Medical Imaging, vol. 22, no. 10, pp. 1235-1251, 2003.

[12] T. Vincent, L. Risser, and P. Ciuciu, "Spatially adaptive mixture modeling for analysis of within-subject fMRI time series," IEEE Trans. on Medical Imaging, vol. 29, no. 4, pp. 1059-1074, 2010. 